\title{
Preparation, characterization and evaluation of fenofibrate: benzoic acid cocrystals with enhanced pharmaceutical properties
}

\author{
Braham Dutt', Manjusha Choudhary ${ }^{2}$ and Vikas Budhwar ${ }^{1 *}$
}

\begin{abstract}
Background: Cocrystallization process involved the understanding of interaction at molecular level between two molecules in context to their crystal packing and designing of new solids having improved physicochemical as well as pharmaceutical properties. In the present research, an attempt to increase the aqueous solubility and dissolution rate of a poorly aqueous soluble drug fenofibrate (FB) by formulation and evaluation of its cocrystals with benzoic acid (BZ) as a coformer was carried out.

Results: The drug and coformer were cocrystallized by using the solvent drop grinding method. For prediction of cocrystals formation, CSD (Cambridge Structure Database) software was utilized. Fourier transformation infrared spectroscopy (FTIR), X-ray diffraction (XRD) and differential scanning calorimetry (DSC) techniques were used for analysis of cocrystals. Albino rats were procured from institution as per IAEC guidelines for in vivo anti-hyperlipidaemic studies. The in vitro dissolution profile of cocrystals, pure drug, their physical mixture and marketed formulation was found to be $89 \%, 39 \%, 47 \%$ and $61 \%$, respectively.

Conclusions: An enhanced anti-hyperlipidaemic activity of cocrystals was found compared to pure drug. The FB: BZ cocrystals also compared to the pure drug showed better dissolution profile and improved in vivo anti-hyperlipidaemic activity in rats. The study proved that cocrystals can promise to improve in vitro dissolution rate of poorly aqueous soluble drugs, which in turn can lead to better in vivo activities.
\end{abstract}

Keywords: Cocrystallization, Cocrystals, Fenofibrate, Multicomponent system, Anti-hyperlipidaemic activity

\section{Background}

The cocrystallization process is long known; however, recently it is gaining much attention because of its wider application in pharmaceuticals as a newer technique to transform the physicochemical properties of drugs like solubility, stability, bioavailability, thermostability and many more. The key advantage in cocrystallization is non-modification of pharmacological activity of drug, while their pharmaceutical properties get modified [1]. The designing of cocrystals depends upon

\footnotetext{
*Correspondence: vikaasbudhwar@yahoo.com

1 Department of Pharmaceutical Sciences, Maharishi Dayanand

University, Rohtak, Haryana 124001, India

Full list of author information is available at the end of the article
}

hydrogen bonding and potential intermolecular interaction between drug and coformer [2]. FB is currently used as anti-hyperlipidaemic drug, which have a direct effect on lowering the cholesterol, triglycerides and LDL (lowdensity lipids), VLDL (very-low-density lipids) along with raising the level of HDL (high-density lipids) in blood. It belongs to the group of drugs called 'fibrate'. The poor aqueous solubility of drug causes low bioavailability and limited permeability through the epithelial membrane [3]. This project work has been designed with purpose to improve the solubility profile of water-insoluble drug $\mathrm{FB}$ through its cocrystallization with $\mathrm{BZ}$ via the solvent drop grinding method. Different techniques like CSD software were applied to select the suitable coformer along with determination of all possibilities of cocrystals 
formation [4]. Drug and coformer in a fixed stoichiometric ratio, viz. 1:1, were grinded in mortar and pestle with dropwise addition of ethanol as solvent [5]. The analysis cocrystals were performed by using FTIR, XRD and DSC methods [6]. The in vitro drug release profile of cocrystals was performed in $0.05 \%$ SLS (sodium lauryl sulphate) medium and compared with pure drug and marketed formulation $[7,8]$. The dissolution study exhibited improved drug release profile of cocrystals (89\%) compared to pure drug (39\%), their physical mixture (47\%) and marketed formulation (61\%), respectively. The in vivo anti-hyperlipidaemic activity of cocrystals were performed by using Albino rats (200-250 g), and the results of study exhibited a higher level of control in serum cholesterol, triglycerides, HDL, LDL and VLDL level in comparison with the FB.

\section{Methods}

FB was obtained as gift sample from Jubilant Generics (Noida). BZ was purchased from Sigma-Aldrich Chemical Limited and Triton X100 from Sigma-Aldrich Chemical Limited. Solvent drop grinding technique was utilized for the preparation of cocrystals. Disease-free animals were procured from animal house as per IAEC guidelines of concerned institution. The study was approved by Institutional Animal Ethics Committee [Reference no. 1767/GO/Re/S/14/CPCSEA; dated: 15/06/2019]. Owners of the animals have given a written consent to the authors to use the animals for experimental work. Twenty-four Albino rats were kept in four groups having 6 rats in each group. Triton was injected at a dose of $(300 \mathrm{mg} / \mathrm{kg})$ intraperitoneal after dissolving it into normal saline to all groups except group I. Control group received intragastrically administration of normal saline, while groups II and III receive intragastrically FB:BZ cocrystals in a dose of $25 \mathrm{mg} / \mathrm{kg}$ and $50 \mathrm{mg} / \mathrm{kg}$, respectively. The last group (IV) receive standard $\mathrm{FB} 50 \mathrm{mg} / \mathrm{kg}$ intragastrically. No anaesthetic and euthanasia methods used in this study. Animals were returned to the institutional animal house after experimentation. Cocrystallization technique mainly involves around synthon formation tendency between functional groups of drug and coformer. Lots of techniques and software like CSD, COSMOS-RS techniques are being used to find out these prone functional groups having hydrogen-bonding tendency. FB structure was evaluated and analysed on CSD by using its CSD code 'TADLIU'. On the basis of CSD software study, no active hydrogen bond donor or acceptor is present in FB molecule. Only halogen $(\mathrm{Cl})$ is active for interaction with other functional groups, while in case of $\mathrm{BZ}$ only $\mathrm{COOH}$ functional group is present, which could make interaction with another functional group. In this study, it was hypothesized that linkage between halogen functional group of FB and carboxylic functional group of BZ could take place via hydrogen bonding. For the preparation of cocrystals, both drug and coformer were used in a fixed stoichiometric ratio (1:1) and ground with the addition of dropwise ethanol. The grinding process was performed manually with the addition of dropwise ethanol in mortar pestle. Cocrystals were procured after complete evaporation of solvent at room temperature. Spectroscopical study was carried out to find out the absorption maxima $\left(\lambda_{\max }\right)$ of FB, which came out to be $229 \mathrm{~nm}$.

\section{In vivo studies}

The animal experiments complied with the ARRIVE guidelines. ARRIVE checklist has been attached as Additional file 1 .

\section{Result}

Characterization of $\mathrm{FB}, \mathrm{BZ}$, their physical mixture and cocrystals

For characterization purposes, FB, BZ, FB:BZ physical mixture and cocrystals were used. For the preparation of physical mixture, both $\mathrm{FB}$ and $\mathrm{BZ}$ were taken in equal amount (100 mg), respectively [9].

\section{DSC analysis}

DSC Q10 (V9.9 Build 303, US instrument) was utilized for DSC analysis of pure drug, coformer, their physical mixture and cocrystals. Two milligrams of the sample was used, and these samples were analysed in a closed aluminium pan, and the temperature range for heating was kept between 20 and $160{ }^{\circ} \mathrm{C}$ at $10{ }^{\circ} \mathrm{C}$ heating rate in the presence of nitrogen gas flow of $60 \mathrm{ml} / \mathrm{min}$. A sharp endothermic peak was obtained for both drug and coformer at $82.64{ }^{\circ} \mathrm{C}$ and $126.44{ }^{\circ} \mathrm{C}$, respectively, which exhibits the purity of procured samples (Fig. 1a, b). In the thermogram of the physical mixture of drug and coformer, the corresponding peaks of drug and coformer are absent, instead a sharp peak at $85.60{ }^{\circ} \mathrm{C}$ shows the physicochemical interaction of drug with coformer when the temperature of their physical mixture in the DSC pans was increased. This conformed the requirement of solvent during the cocrystallization process. In the DSC thermogram of cocrystals, no other endothermic peaks were present in spite of a sharp endothermic peak at $85.01{ }^{\circ} \mathrm{C}$, which conforms that both drug and coformer have been completely cocrystallized (Fig. 1d).

\section{FTIR analysis}

FTIR (Alpha Bruker 1206 0280, Germany instrument) was utilized for FTIR study of FB, BZ, their physical mixture and cocrystals by using the $\mathrm{KBr}$ disc method. For this analysis, the range of spectrum recording was kept between 4000 and $400 \mathrm{~cm}^{-1}$. The characteristic 

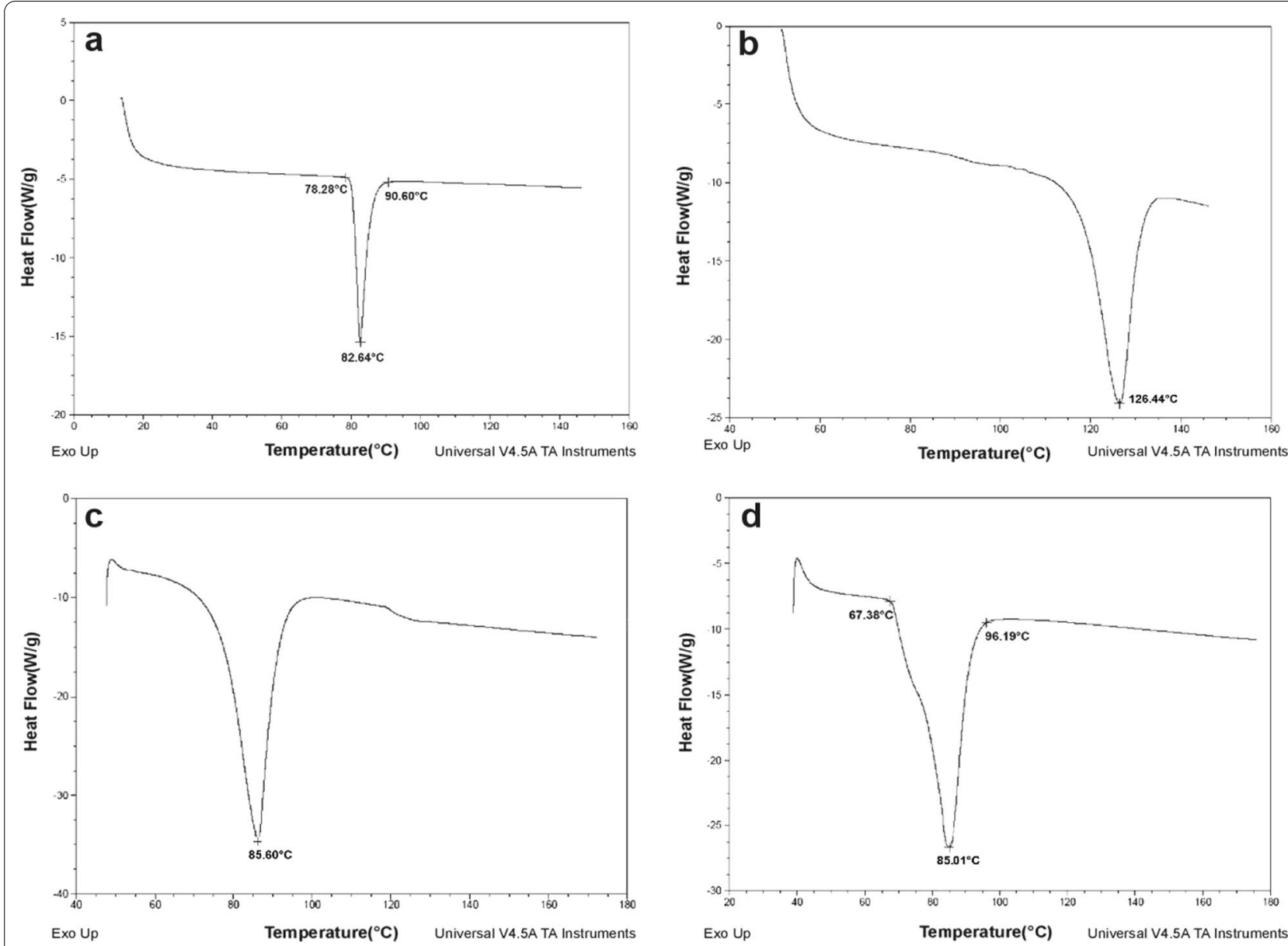

Fig. 1 DSC thermogram of a FB, $\mathbf{b} B Z, \mathbf{c}$ physical mixture and $\mathbf{d}$ cocrystals

peaks of $\mathrm{FB}, \mathrm{BZ}$, their physical mixture and cocrystals as shown in Fig. 2 are explained in Table 1, respectively. The characteristic peaks present in FTIR spectra of FB, viz. C-H stretch (aromatic) at $2984 \mathrm{~cm}^{-1}$, at $1288 \mathrm{~cm}^{-1}$ and $1246 \mathrm{~cm}^{-1}$ for C-O stretch (arylkyl) and $1648 \mathrm{~cm}^{-1}$ for $\mathrm{C}=\mathrm{O}$ functional group and $\mathrm{C}-\mathrm{O}$ stretch at $1091 \mathrm{~cm}^{-1}$ along with a halogen compound (chlorine) at $761 \mathrm{~cm}^{-1}$. In case of $\mathrm{BZ} \mathrm{C}-\mathrm{O}$ stretch (acidic) at $1279 \mathrm{~cm}^{-1}$ for carboxylic acid functional group and at $1684 \mathrm{~cm}^{-1}$ for $\mathrm{C}=\mathrm{O}$ stretch (carbonyl) were found, respectively. The characteristic peak between 3609 and $3794 \mathrm{~cm}^{-1}$ for $\mathrm{O}-\mathrm{H}$ (free) was observed. Most of these characteristic peaks were also present in their physical mixture with minor peak shifting due to some weak van der Waals forces, viz. $1279 \mathrm{~cm}^{-1}$ for C-O stretch, $1648 \mathrm{~cm}^{-1}$ for $\mathrm{C}=\mathrm{O}$ stretch and $2992 \mathrm{~cm}^{-1}$ for $\mathrm{C}-\mathrm{O}$ stretch $^{3}$. The absence of $\mathrm{C}-\mathrm{O}$ stretch in spectrum of cocrystals at $2992 \mathrm{~cm}^{-1}$, which was present in physical mixture of both drug and coformer along with shifting of $\mathrm{C}=\mathrm{O}$ stretch (carbonyl functional group) for $\mathrm{BZ}$ at $1684 \mathrm{~cm}^{-1}$ and $\mathrm{FB}$ at $1648-1702 \mathrm{~cm}^{-1}$ conformed interaction between drug and coformer [10].

\section{XRD analysis}

XRD analysis of FB, BZ, FB: BZ physical mixture and cocrystals was performed by using XRD model 'XPERT PRO' instrument with continuous scanning type at $2 \theta$ angle position (Fig. 3). The XRD spectrum of FB exhibited characteristic peaks at 11.24, 11.86, 14.34, 16.14, $16.61,19.18,22.17$ and 26.14 having relative intensity of 13.48, 19.80, 44.85, 71.11, 58.71, 32.28, 100 and 32.39, respectively. The XRD spectrum of $\mathrm{BZ}$ exhibited characteristic peaks at 7.9682, 8.1026, 16.1232 and 17.1394 having relative intensity of $72.62,85.34,62.71$ and 100.00 , respectively. The XRD spectrum of physical mixture of FB:BZ exhibited characteristic peaks at 8.13, 16.27, 17.20, $19.15,22.20,30.12$ and 32.89 having relative intensity of 100.00, 35.64,6. 2.36, 6.86, 1.40 and 2.54, respectively. The XRD spectrum of cocrystals exhibited characteristic peaks at $6.27,8.11,11.26,11.85,14.33,15.68,16.60,20.80$, 

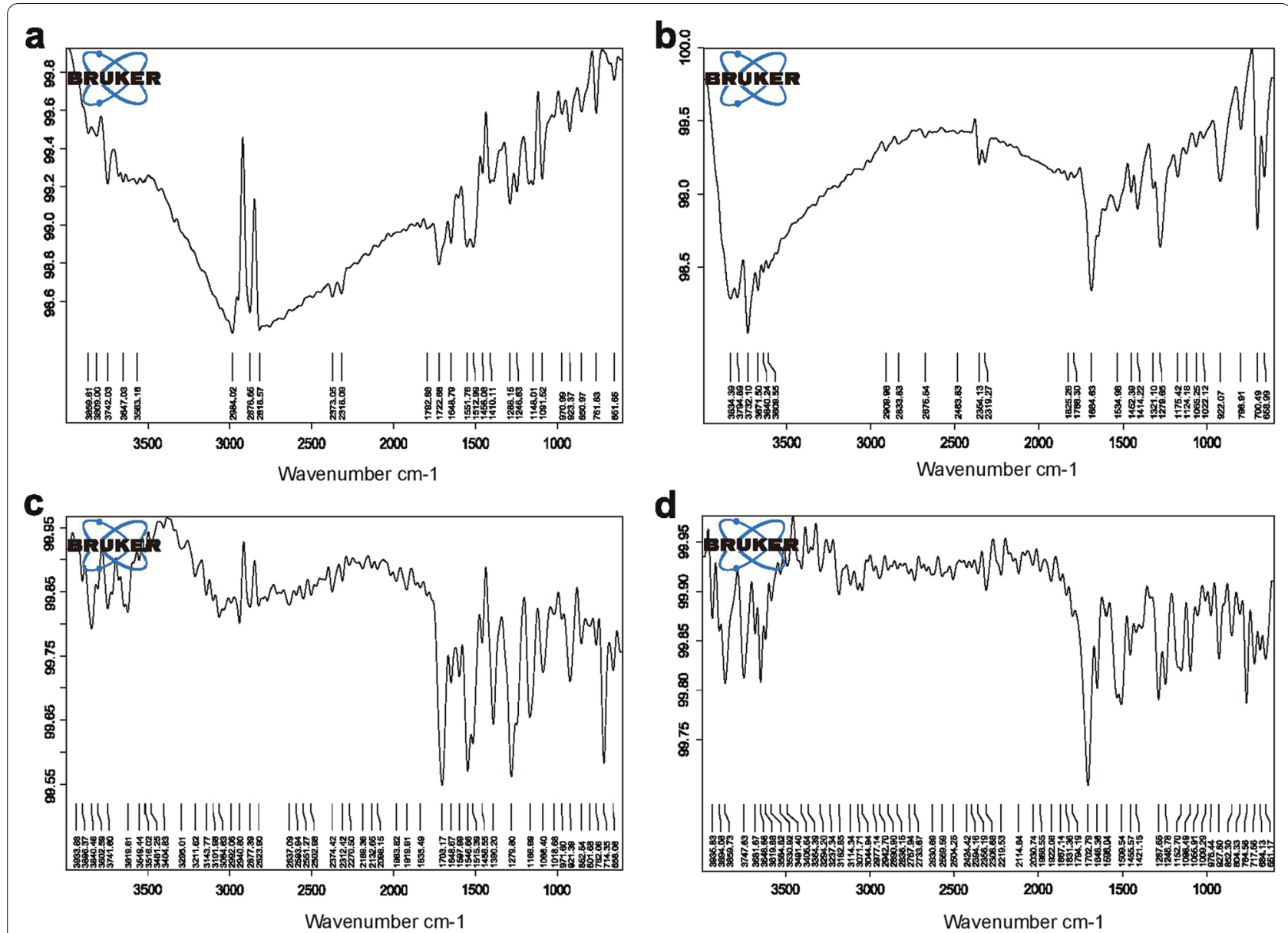

Fig. 2 FTIR spectrum of a FB, $\mathbf{b} B Z$, $\mathbf{c}$ physical mixture and $\mathbf{d}$ cocrystals

Table 1 FTIR data of FB, BZ, their physical mixture and cocrystals

\begin{tabular}{lllll}
\hline Chemical bond & FB & BZ & Physical mixture & Cocrystals \\
\hline C-H stretch & $\begin{array}{l}2876,2984 \text { (aliphatic and } \\
\text { aromatic) }\end{array}$ & & \\
C=O stretch & 1648 & 1684 (Carbonyl) & 1648 (Carbonyl) & 1702 (Carbonyl) \\
C-O stretch & $1246-1288$ (Arylkyl) & $1175-1279$ (Acidic) & 2992 (Arylkyl) & 1287 (Arylkyl) \\
& & & 1279 (Acidic) & 1098 (Dialkyl ether) \\
C-O stretch & 1091 (Dialkyl ether) & & 762 (Halogen) & \\
Cl (Short) & 761 (Halogen) & 3609-3794 (Alcohol) & & 764 (acid-halogen interaction) \\
O-H Stretch & & & & \\
Cl-H (Medium) & & &
\end{tabular}

$22.15,30.58$ and 38.77 having relative intensity of 13.36 , $100,24.56,51.20,63.27,59.28,53.28,88.91,81.94,69.98$ and 43.55 , respectively. In case of eutectic mixtures, the height of characteristic peaks did not much change, but in case of cocrystals newer peaks get generated, which is evident in the XRD spectrum. But here, the generation of newer characteristic peaks conformed the formation of newer crystalline structure. On the basis of FTIR data and XRD data, it is confirmed that a newer crystalline compound has been generated with improved pharmaceutical and physicochemical properties compared to parent components. The linkage between - $\mathrm{COOH}$ functional group of coformer and halogen functional group of drug takes place. 

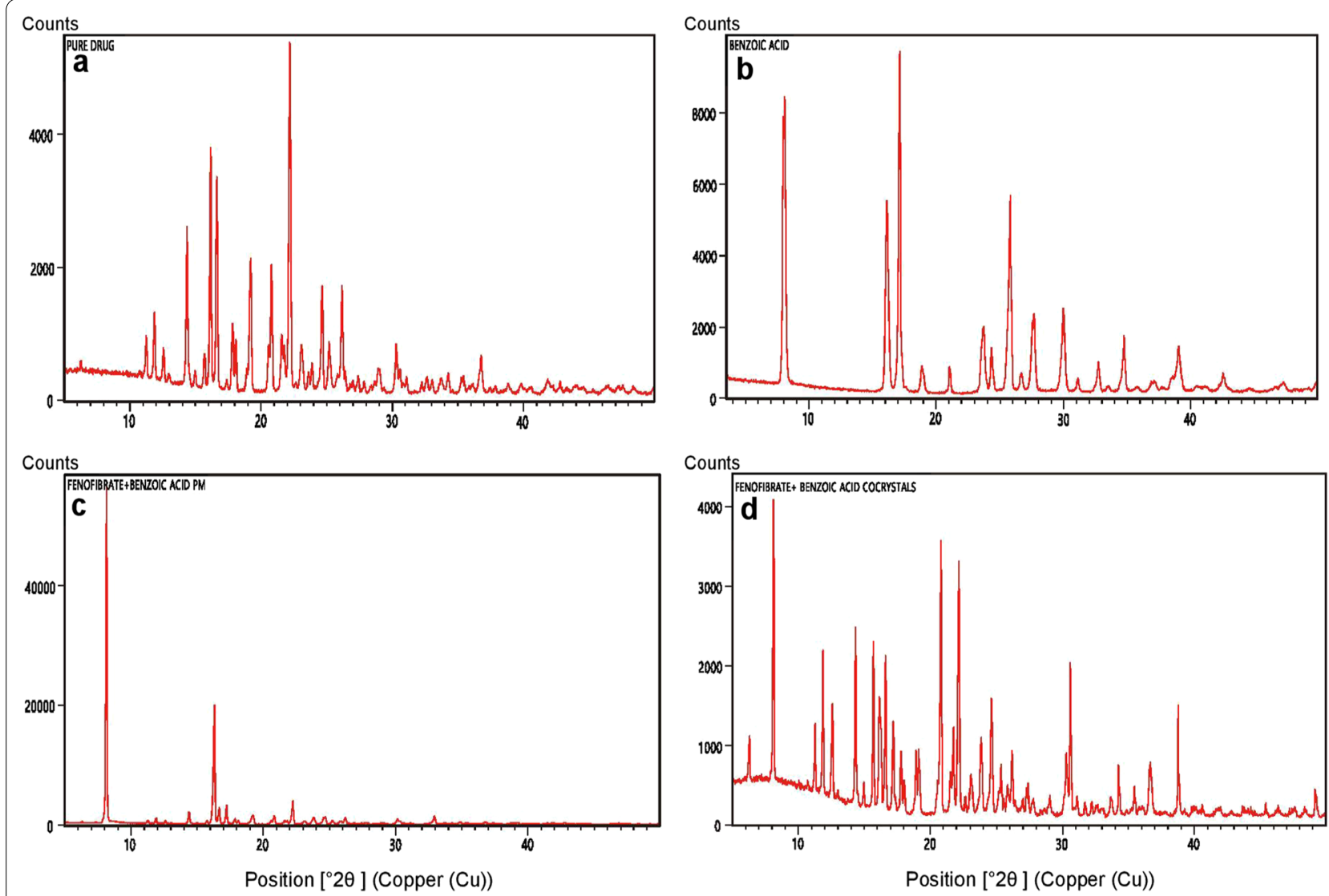

Fig. 3 XRD spectrum of a FB, $\mathbf{b} B Z$, c physical mixture and $\mathbf{d}$ cocrystals

\section{In Vitro drug release study}

For the dissolution study, USP Type II dissolution apparatus (Lab India DS-8000) was used. Forty milligrams of pure FB was used for this study [11]. 0.05\% SLS solution was used as dissolution medium for studying the drug release profile of pure drug, marketed formulation and FB:BZ physical mixture. The paddle speed was kept at $50 \mathrm{rpm}$ on $37 \pm 0.5{ }^{\circ} \mathrm{C}$ temperature for $30 \mathrm{~min}$ [7]. Samples $(5 \mathrm{ml})$ were withdrawn and suitable for dilution for spectroscopical evaluation. The dissolution study revealed improved drug release profile of cocrystals compared to pure drug, marketed formulation and FB:BZ physical mixture, respectively. Pure FB exhibited approx. $39 \%$ drug release, while marketed formulation, cocrystals and FB:BZ physical mixture exhibited $61 \%$, $89 \%$ and $47 \%$ drug release, respectively, over 30 -min period (Fig. 4).

\section{In Vivo triton-induced anti-hyperlipidaemic study}

For in vivo anti-hyperlipidaemic study, Albino rats were starved overnight. The animals were maintained in polypropylene cages in a well-ventilated room, at temperature of $25 \pm 1{ }^{\circ} \mathrm{C}$ with 12:12-h light/dark cycle. Rats were kept

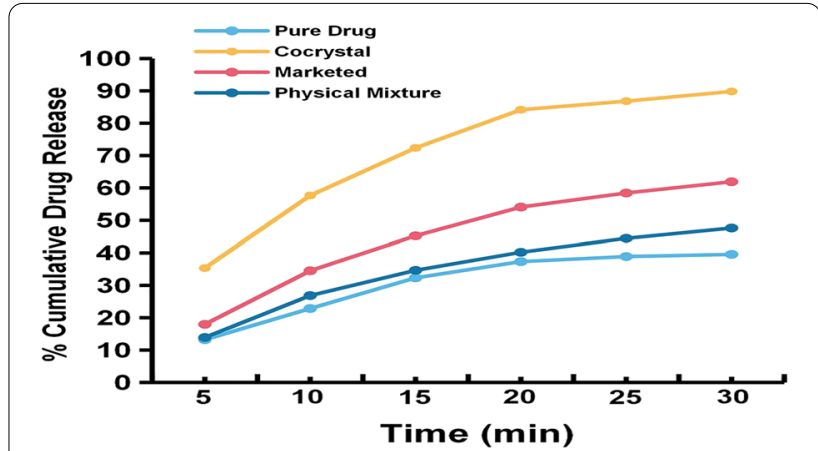

Fig. 4 Comparison of drug release study, pure drug exhibited 39\% drug release while marketed formulation, cocrystals and physical mixture exhibited $61 \%, 89 \%$ and $47 \%$ drug release, respectively, over 30-min period

in four groups having six rats in each group. Triton was injected at a dose of $(300 \mathrm{mg} / \mathrm{kg})$ intraperitoneal after dissolving it into normal saline to all groups except group I [11]. Control group received intragastrically administration of normal saline, while groups II and III receive intragastrically FB:BZ cocrystals in a dose of $25 \mathrm{mg} / \mathrm{kg}$ 
and $50 \mathrm{mg} / \mathrm{kg}$, respectively. The last group (IV) receive standard FB $50 \mathrm{mg} / \mathrm{kg}$ intragastrically (Table 2).

At $18 \mathrm{~h}$ following triton administration, blood samples were collected from the tail vein and immediately centrifuged [12, 13]. Finally, serum lipid profile was assayed using standard diagnostic kits. The anti-hyperlipidaemic effects of FB and BZ cocrystals on triton-induced antihyperlipidaemic rat model are presented in Table 3 . The dose of cocrystals in group II was kept half compared to standard drug FB (group IV) and in group III equivalent dose of cocrystals to the standard drug was used (Table 3). There was a gradual increase in serum lipids of rats in the control group. However, in the FB group (50 mg/kg), half dose of cocrystals $(25 \mathrm{mg} / \mathrm{kg})$ and full dose of cocrystals group $(50 \mathrm{mg} / \mathrm{kg})$ showed a significant reduction in the serum lipids profile (Table 3).

\section{Discussion}

In the DSC thermogram of the cocrystals and physical mixture of drug and coformer, the absence of corresponding endothermic peaks of FB and BZ conformed the complete cocrystallization of drug and coformer. The presence of single endothermic peak in physical mixture was due to cocrystallization of FB:BZ physical mixture due to increased temperature of DSC pan. Due to heating of samples, the moisture content present in the drug and coformer acts as solvent and leads to automatic cocrystallization of drug and conformer, so no trace of extra amount remained non-cocrystallized, that's why only single endothermic peak was obtained. The onset temperature for melting of cocrystals is $67.30{ }^{\circ} \mathrm{C}$ and

Table 2 In vivo anti-hyperlipidaemic study

\begin{tabular}{lllll}
\hline S. No. & Group & Treatment & Dose $\mathbf{( m g / k g )}$ & $\begin{array}{l}\text { Route of } \\
\text { administration }\end{array}$ \\
\hline 1 & I & Triton & 300 & $\begin{array}{l}\text { Injected intra- } \\
\text { peritoneal }\end{array}$ \\
2 & II & FB:BZ cocrystals & 25 & Oral route \\
3 & III & FB:BZ cocrystals & 50 & Oral route \\
4 & IV & FB & 50 & Oral route \\
\hline
\end{tabular}

${ }^{*}$ Total number of rats $=24$
$71.12{ }^{\circ} \mathrm{C}$ for physical mixture. In the FTIR analysis, corresponding characteristic peaks of $\mathrm{FB}$ and $\mathrm{BZ}$ were present in physical mixture with minor shifting. There was shifting of some characteristic peaks in 1:1 physical mixture of FB:BZ, which might be due to weak van der Waals interactions between FB and BZ. This confirmed that there was no physicochemical interaction between drug and coformer without the addition of any solvent or liquid medium. Most of these characteristic peaks were also present in their physical mixture with minor peak shifting, viz. $1279 \mathrm{~cm}^{-1}$ for $\mathrm{C}-\mathrm{O}$ stretch, $1648 \mathrm{~cm}^{-1}$ for $\mathrm{C}=\mathrm{O}$ stretch and $2992 \mathrm{~cm}^{-1}$ for $\mathrm{C}-\mathrm{O}$ stretch. The hydrogen bonding was conformed by the absence of $\mathrm{OH}$ stretch present in BZ spectrum along with shifting of characteristic peak of halogen functional group [3] from 761 to $764 \mathrm{~cm}^{-1}$. In case of XRD analysis, there was minor shifting of characteristic XRD peaks of physical mixture due to minor interactions of $\mathrm{FB}$ and $\mathrm{BZ}$ molecules during mixing process [3]. Due to hydrogen bonding between carboxylic acid of coformer and halogen functional group [14-16] of drug, newer crystal structure has been generated with different crystalline properties. The dissolution study exhibited an increased drug release profile of cocrystals in comparison with the FB, marketed formulation and FB:BZ physical mixture [17]. According to the outcomes of drug release profile study, it was concluded that solubility profile of BCS class-II drugs could be modified through cocrystallization process. In vivo anti-hyperlipidaemic study of cocrystals exhibited the effect in a dose-dependent manner. There was no significant reduction in serum lipids profile found in case of group I rats treated with saline. The results revealed that cocrystals with $50 \mathrm{mg} / \mathrm{kg}$ dose [18] significantly reduced the serum lipids followed by standard anti-hyperlipidaemic drug, FB $50 \mathrm{mg} / \mathrm{kg}$ (Table 3). Herein, FB and BZ cocrystals have been prepared successfully in fixed stoichiometric ratio (1:1) by using the solvent drop grinding technique [19].

\section{Conclusions}

In this study, FB and BZ have been cocrystallized in fixed stoichiometric ratio (1:1) by using the solvent drop grinding technique. The cocrystals exhibited an

Table 3 Serum lipidaemic data

\begin{tabular}{llrrrrr}
\hline Groups & Dose & TC & TG & HDL & LDL & VLDL \\
\hline I & Control (Triton X100-300 mg/kg) & $173.05 \pm 3.89$ & $169.48 \pm 4.46$ & $29.47 \pm 4.53$ & $109.33 \pm 2.89$ & $28.86 \pm 3.49$ \\
II & Cocrystals (25 mg/kg) & $159.67 \pm 4.85$ & $122.31 \pm 3.82$ & $27.80 \pm 0.59$ & $102.10 \pm 2.31$ & $26.73 \pm 1.41$ \\
III & Cocrystals (50 mg/kg) & $89.23 \pm 3.72$ & $81.37 \pm 3.35$ & $38.12 \pm 1.13$ & $45.97 \pm 2.09$ & $15.83 \pm 0.98$ \\
IV & FB (50 mg/kg) & $108.2 \pm 2.55$ & $98.55 \pm 2.03$ & $33.21 \pm 1.98$ & $58.31 \pm 3.61$ & $20.76 \pm 2.39$ \\
\hline
\end{tabular}

Values are mean of $6 \pm$ S.D, TC—-total cholesterol, TG—-triglycerides, HDL—high-density lipids, LDL—low-density lipids, VLDL—very-low-density lipids 
improved dissolution profile and enhanced in vivo triton-induced anti-hyperlipidaemic rat model based on anti-hyperlipidaemic activity compared to parent pure drug. Further, stability studies, bioavailability studies, toxicological studies, clinical and preclinical testing are required to commercially exploit the advantages of cocrystallization process.

\section{Abbreviations}

BZ: Benzoic acid; CSD: Cambridge Structure Database; DSC: Differential scanning calorimetry; FB: Fenofibrate; FTIR: Fourier transformation infrared spectroscopy; XRD: X-ray diffraction.

\section{Supplementary Information}

The online version contains supplementary material available at https://doi. org/10.1186/s43094-021-00320-5.

Additional file 1. ARRIVE checklist.

\section{Acknowledgements}

Authors want to acknowledge laboratory and Internet facilities provided by the Department of Pharmaceutical Sciences, Maharishi Dayanand University, Rohtak, India, to complete this study.

\section{Authors' contributions}

BD conducted all the experimentation, writing and editing work. MC supervised all the in vivo anti-hyperlipidaemic studies. VB supervised all the in vitro work including dissolution studies and interpretation of analytical data. All authors read and approved the final manuscript.

\section{Funding}

Not applicable.

\section{Availability of data and material}

Data and material are available upon request.

\section{Declarations}

\section{Ethics approval and consent to participate}

The animal experiments were designed according to the approved animals' ethical committee and related recommendations for performing experiments on animals. The study was approved by Institutional Animal Ethics Committee of Maharishi Dayanand University, Rohtak, India [Reference no. 1767/ GO/Re/S/14/CPCSEA; dated: 15/06/2019]. Owners of the animals have given a written consent to the authors to use the animals for experimental work. Informed consent was confirmed by the IRB. The authors do not claim to contain any content comprising of any study on human subject.

\section{Consent for publication}

Not applicable.

\section{Competing interests}

Authors declare that there is no competing interest.

\section{Author details}

'Department of Pharmaceutical Sciences, Maharishi Dayanand University, Rohtak, Haryana 124001, India. ${ }^{2}$ University Institute of Pharmaceutical Sciences, Kurukshetra University, Kurukshetra 136119, India.

Received: 5 May 2021 Accepted: 10 Auqust 2021

Published online: 26 August 2021

\section{References}

1. Alhalaweh A, Sokolowski A, Rodríguez-Hornedo N, Velaga SP (2011) Solubility behavior and solution chemistry of indomethacin cocrystals in organic solvents. Cryst Growth Des 11:3923-3929. https://doi.org/10.1021/cg200 $517 r$

2. Fayos I (2009) Molecular crystal prediction approach by molecular similarity: data mining on molecular aggregation predictors and crystal descriptors. Cryst Growth Des 9(7):455-465. https://doi.org/10.1021/cg801122m

3. Gaikwad ER, Khabade SS, Sutar TB, Payghan SA (2017) Preparation and characterization of molecular complexes of fenofibrate cocrystal. Asian J Pharm 11(4):746-753

4. Qiao N, Li M, Schlindwein W, Malek N, Davies A, Trappitt G (2011) Pharmaceutical cocrystals: an overview. Int J Pharm 419:1-11. https://doi.org/10. 1016/j.jpharm.2011.07.037

5. Cuadra IA, Cabañas A, Chedaa JAR, Martínez-Casadob FJ, Pando C (2016) Pharmaceutical co-crystals of the anti-inflammatory drug diflunisal and nicotinamide obtained using supercritical $\mathrm{CO}_{2}$ as an antisolvent. J $\mathrm{CO} 2 \mathrm{Util}$ 13:29-37. https://doi.org/10.1016/j.jcou.2015.11.006

6. Hisada N, Takano R, Takata N, Shiraki K, Ueto T, Tanida S et al (2016) Characterizing the dissolution profiles of supersaturable salts, cocrystals, and solvates to enhance in vivo oral absorption. Eur J Pharm Biopharm 103:192-199. https://doi.org/10.1016/j.ejpb.2016.04.004

7. United States Pharmacopeia and National Formulary (2016) Rockville, MD: United States Pharmacopeial Convention.

8. Raghuram M, Alam MS, Prasad M, Khanduri CH (2016) Pharmaceutical cocrystal of prulifloxacin with nicotinamide. Int J Pharm Pharm Sci 6(10):180-184

9. Yamashita H, Hirakura Y, Yuda M, Teramura T, Terada K (2013) Detection of cocrystal formation based on binary phase diagrams using thermal analysis. Pharm Res 30:70-80. https://doi.org/10.1007/s1 1095-012-0850-1

10. Jijith US, Jayakumari S (2018) Screening methods for antihyperlipidemic activity: a review. Drug Invent Today 10(2):257-259

11. Shewale S, Shete AS, Doijad RC, Kadam SS, Patil VA, Yadav AV (2015) Formulation and solid-state characterization of nicotinamide-based co-crystals of fenofibrate. Indian J Pharm Sci 77(3):328-334

12. Hikmat S, Al-qirim T, Alkabbani D et al (2017) Synthesis and in vivo antihyperlipidemic activity of novel n-benzoylphenyl-2-furamide derivatives in Wistar rats. Trop J Pharm Res 16(1):193-201

13. Cheney ML, Weyna DR, Shan N, Hanna M, Wojtas L, Zaworotko MJ (2011) Coformer selection in pharmaceutical cocrystal development: a case study of a meloxicam aspirin cocrystal that exhibits enhanced solubility and pharmacokinetics. J Pharm Sci 6(100):2172-2181. https://doi.org/10.1002/ jps.22434

14. Allen FH (2002) The Cambridge structural database: a quarter of a million crystal structures and rising. Acta Cryst 58:380-388. https://doi.org/10.1107/ S0108768102003890

15. Blagden N, Coles SJ, Berry DJ (2014) Pharmaceutical co-crystals—are we there yet? Cryst Eng Comm 16:5753. https://doi.org/10.1039/c4ce00127c

16. Lemmerer A, Govindraju S, Johnston M, Motloung X, Savig KL (2015) Cocrystals and molecular salts of carboxylic acid/ pyridine complexes: can calculated pKa's predict proton transfer? A case study of nine complexes. CrystEngComm 17:3591. https://doi.org/10.1039/c5ce00102a

17. Code for Fenofibrate on CSD software was accessed by using the link https://www.ccdc.cam.ac.uk/structures/Search?Compound=fenofibrate\& DatabaseToSearch=Published. Accessed on 07 April 2018

18. Kumar S, Nanda A (2017) Pharmaceutical cocrystals: an overview. Indian J Pharm Sci 79(6):858-871. https://doi.org/10.4172/pharmaceutical-sciences. 1000302

19. Yamashita H, Hirakura Y, Yuda M, Teramura T, Terada K (2017) Detection of cocrystal formation based on binary phase diagrams using thermal analysis. Pharm Res 30:70-80. https://doi.org/10.1007/s1 1095-012-0850-1

\section{Publisher's Note}

Springer Nature remains neutral with regard to jurisdictional claims in published maps and institutional affiliations. 